carefully study the changes which take place in the vitreous as the result of the development of fibrillar streamers from the region of an operative trauma. In cataract work the eventual test of such vitreous changes is of course the vision, and it must be confessed that many cases in which there is considerable vitreous change on microscopic and ophthalmoscopic investigation, preserve fairly good vision for a considerable time. Strictly speaking, the result of an operation for cataract ought to be recorded at least twelve months after the section. In this way only will the truth be arrived at as to the permanent and lasting value of any method. This is a very difficult matter in India, but no difficulty ought to be experienced in gradually collecting large figures in other countries where hospital cases are more easily kept in view. If only the perfect "operative" and "immediate post-operative" results were followed up the figures would be strictly comparable. In making these statements I do not lose sight of the fact that it is better for a patient to have vitreous opacities and a gradually darkening eye than not to have vision at all. The danger of the development of vitreous opacities after removing the lens in its capsule may not be so important as would appear from the foregoing when applied to selected eyes, healthy except for cataract, such as are usually chosen for operation in Western clinics, but even then one has to consider the other effects of leaving the vitreous unsupported, as suggested at the beginning of this note. Time and the accurate records of carefully followed cases wilı tell.

\title{
A NOTE ON PULFRICH'S PHENOMENON WITH A SUGGESTION ON ITS POSSIBLE CLINICAL IMPORTANCE
}

BY

\author{
H. GRIMSDale \\ LONDON
}

ABout eighteen months ago, Mr. J. H: Tomlinson drew my attention to the curious phenomenon which Pulfrich had recently described, and gave me a small model on which the phenomenon could be demonstrated.

It will be remembered that Pulfrich had noted the fact that if one eye has its light sense diminished by covering with a dark glass, the image from this eye is recognized by the brain more slowly than the image from the normal eye. 
This is demonstrated by means of two pointers which move in a plane; when observed by two eyes whose light sense is equal, it is seen that the two pointers are in one plane, but when the light sense of one is diminished, the moving pointer appears to revolve round the other. The explanation is that the recognition of a

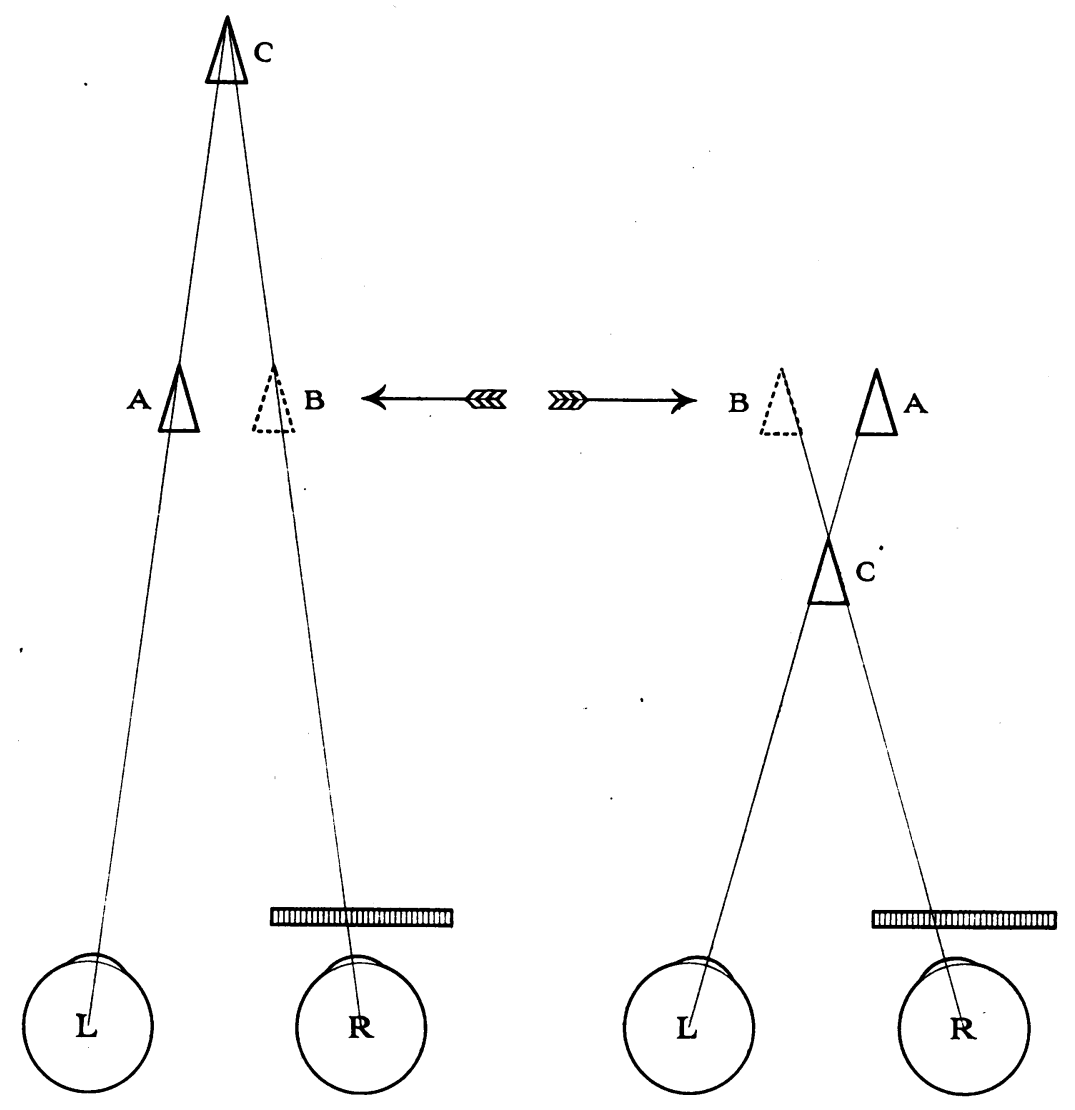

stimulus varies in time, with the intensity; a lesser stimulus requires a longer time. Therefore, though the eye which is covered with a dark glass sees an object moving at the same rate as the unaffected eye, the movement appears to begin rather later, and the object is "seen" behind its true position, that is to say the brain receives the impression from the object at a given point but takes longer to interpret it.

Two diagrams will make the apparent movement clear. The direction of movement is shown by the arrow. 
In the diagrams $\mathrm{R}$ is the right eye behind the dark glass, $\mathrm{L}$ the left eye; A the object as seen by the left (normal) eye, B its image as seen from the right eye. $\mathrm{C}$ is the image in the faulty position.

I do not think that it has been pointed out that this phenomenon has a definite limited clinical importance; we are, as ophthalmic surgeons, not infrequently consulted by patients who find difficulty in games; men, getting on in years, who have been expert in games with a moving ball, find themselves unable to judge its position with their former accuracy, and, therefore, do not meet the ball with the middle of the bat. Pulfrich's phenomenon seems to give a probable explanation ; at least in a considerable proportion of cases.

Not only will the normal loss of transparency of the lens diminish the acuity of the light sense and therefore lengthen the time reaction, but also, if one eye has any change specially diminishing its acuity, there will be an alteration of the position of the ball varying with the angle at which it approaches the striker.

I had suspected that this was the chief difficulty in a number of patients but had not found one which appeared to prove it, until recently; however, a few weeks ago I was consulted by a young man who had been a good tennis player, on account of this difficulty; that the balls seemed sometimes too near and sometimes too far; on going into his history, I found that he had had some three months before, an attack of retro-ocular neuritis. From this he had recovered well; his acuity was $6 / 9$ all, in the affected eye; but when I tested him, the light sense was manifestly lessened, and with Pulfrich's model, the points seemed constantly to revolve round one another.

I found on further examination, that a No. 2 smoke, placed in front of the good eye stopped this apparent movement; and I, therefore, suggested to him that it might be possible to correct some of the disability by wearing a dark glass over the good eye.

$\mathrm{He}$ promised to let me know how this arrangement worked; but so far I have not heard.

It is clear that the image perception being delayed in both eyes there will be a constant mal-appreciation of the true position; but a constant error is much more easily corrected mentally than one which is always varying.

It seems to me probable that the loss of transparency which constantly comes on, in the lens, with advance of years, is the cause, through this delayed recognition, of much of the loss of accurate timing in games with a moving ball. 